\title{
Hausdorff dimension of the contours of symmetric additive Lévy processes
}

\author{
Davar Khoshnevisan • Narn-Rueih Shieh • \\ Yimin Xiao
}

Received: 22 September 2008 / Revised: 22 September 2008 / Published online: 8 November 2008 (C) Springer-Verlag 2008

\section{Erratum to: Probab. Theory Relat. Fields (2008) 140:129-167 DOI 10.1007/s00440-007-0060-7}

The last portion of the proof of Theorem 3.2 (pp. 161-162) in [1] contains a deceptively subtle logical flaw: the proof correctly establishes that the lower bound for the Hausdorff dimension $\operatorname{dim}_{\mathrm{H}}\left(\mathfrak{X}^{-1}(\{0\}) \cap F\right)$ holds with positive probability; but from that we incorrectly deduced that the said Hausdorff-dimension bound holds almost surely on $\left\{\mathfrak{X}^{-1}(\{0\}) \cap F \neq \varnothing\right\}$. To correct this, the statements of some of the results need to be altered slightly.

The online version of the original article can be found under doi:10.1007/s00440-007-0060-7.

D. Khoshnevisan $(\varangle)$

Department of Mathematics, The University of Utah,

155 S.1400 E, Salt Lake City, UT 84112-0090, USA

e-mail: davar@math.utah.edu

URL: http://www.math.utah.edu/ davar

N.-R. Shieh

Department of Mathematics, National Taiwan University,

Taipei 10617, Taiwan

e-mail: shiehnr@math.ntu.edu.tw

Y. Xiao

Department of Statistics and Probability, Michigan State University,

A-413 Wells Hall, East Lansing, MI 48824, USA

e-mail:xiao@stt.msu.edu

URL: http://www.stt.msu.edu/ xiaoyimi 
- (1.9) should be replaced by the following:

$\operatorname{dim}_{\mathrm{H}} \mathfrak{X}^{-1}(\{0\})=\sup \left\{q>0: \int_{[0,1]^{N}} \frac{\Phi(\boldsymbol{t})}{\|\boldsymbol{t}\|^{q}} \mathrm{~d} \boldsymbol{t}<\infty\right\}$ with positive probability.

This follows from [2, Theorem 2.10] and [1, (5.22)].

- (3.14) should be replaced by

$$
\begin{aligned}
& \left\|\operatorname{dim}_{\mathrm{H}}\left(\mathfrak{X}^{-1}(\{0\}) \cap F\right)\right\|_{L^{\infty}(\mathrm{P})} \\
& =\sup \left\{0<q<N: I_{\Phi}^{(q)}(\mu)<\infty \text { for some } \mu \in \mathcal{P}(F)\right\} .
\end{aligned}
$$

- The lower bound in (3.16) should be replaced by

$$
\operatorname{dim}_{\mathrm{H}} F-\overline{\operatorname{ind}}(\Phi) \leq\left\|\operatorname{dim}_{\mathrm{H}}\left(\mathfrak{X}^{-1}(\{0\}) \cap F\right)\right\|_{L^{\infty}(\mathrm{P})} .
$$

- (3.20) and (3.21) should be replaced, respectively, by

$$
\left\|\operatorname{dim}_{\mathrm{H}}\left(\mathfrak{X}^{-1}(\{0\}) \cap F\right)\right\|_{L^{\infty}(\mathrm{P})}=\operatorname{dim}_{\mathrm{H}} F-\sum_{k=1}^{d} \frac{1}{\alpha_{k}}
$$

and

$$
\left\|\operatorname{dim}_{\mathrm{H}}\left(\mathfrak{X}^{-1}(\{0\}) \cap F\right)\right\|_{L^{\infty}(\mathrm{P})}=\operatorname{dim}_{\mathrm{H}} F-\frac{d}{\alpha} .
$$

- (3.24) should be replaced by the following:

$$
\operatorname{dim}_{\mathrm{H}} \mathfrak{X}^{-1}(\{0\})=N-k(\boldsymbol{\alpha})+\frac{\sum_{1 \leq j \leq k(\boldsymbol{\alpha})} \alpha_{j}-d}{\alpha_{k(\boldsymbol{\alpha})}} \text { with positive probability. }
$$

The derivations remain.

\section{References}

1. Khoshnevisan, D., Shieh, N.-R., Xiao, Y.: Hausdorff dimension of the contours of symmetric additive Lévy processes. Probab. Theory Relat. Fields 140, 129-167 (2008)

2. Khoshnevisan, D., Xiao, Y.: Level sets of additive Lévy processes. Ann. Probab. 30, 62-100 (2002) 